\title{
Gravitational Bremsstrahlung and Hidden Supersymmetry of Spinning Bodies
}

\author{
Gustav Uhre Jakobsen, ${ }^{1,2, *}$ Gustav Mogull@, ${ }^{1,2, \dagger}$ Jan Plefka $\oplus^{1, \$}$ and Jan Steinhoff ${ }^{2,}$ \\ ${ }^{1}$ Institut für Physik und IRIS Adlershof, Humboldt-Universität zu Berlin, Zum Großen Windkanal 2, 12489 Berlin, Germany \\ ${ }^{2}$ Max Planck Institute for Gravitational Physics (Albert Einstein Institute), Am Mühlenberg 1, 14476 Potsdam, Germany
}

(Received 1 July 2021; accepted 3 December 2021; published 4 January 2022)

\begin{abstract}
The recently established formalism of a worldline quantum field theory, which describes the classical scattering of massive bodies (black holes, neutron stars, or stars) in Einstein gravity, is generalized up to quadratic order in spin, revealing an alternative $\mathcal{N}=2$ supersymmetric description of the symmetries inherent in spinning bodies. The far-field time domain waveform of the gravitational waves produced in such a spinning encounter is computed at leading order in the post-Minkowskian (weak field, but generic velocity) expansion, and exhibits this supersymmetry. From the waveform we extract the leading-order total radiated angular momentum in a generic reference frame, and the total radiated energy in the center-ofmass frame to leading order in a low-velocity approximation.
\end{abstract}

DOI: 10.1103/PhysRevLett.128.011101

The rise of gravitational wave (GW) astronomy [1] offers new paths to explore our universe, including black hole (BH) population and formation studies [2], tests of gravity in the strong-field regime [3], measurements of the Hubble constant [4], and investigations of strongly interacting matter inside neutron stars [5]. This form of astronomy relies heavily on Bayesian methods to infer probability distributions for theoretical GW predictions (templates), depending on a source's parameters, to match the measured strain on detectors. With the network of GW observatories steadily increasing in sensitivity [6], theoretical GW predictions need to keep pace with the accuracy requirements placed on templates [7]. For the inspiral and merger phases of a binary an important strategy is to synergistically combine approximate and numerical relativity predictions [8], each applicable only to a corner of the parameter space [9].

In this Letter we calculate gravitational waveforms- the primary observables of GW detectors-produced in the parameter-space region of highly eccentric (scattering) spinning BHs and neutron stars, to leading order in the weak-field, or post-Minkowskian (PM), approximation. Following the above strategy, this is a valuable input for future eccentric waveform models. Indeed, the extension of contemporary quasicircular (noneccentric) waveform models for spinning binaries to eccentric orbits (including scattering) is under active investigation [10]. This is motivated, for instance, by the potential insight gained on the formation channels or astrophysical environments of binary BHs (BBHs) through

Published by the American Physical Society under the terms of the Creative Commons Attribution 4.0 International license. Further distribution of this work must maintain attribution to the author(s) and the published article's title, journal citation, and DOI. Funded by SCOAP . measurements of eccentricity [11] and spins [12], or the search for scattering BHs [13] in our universe.

Accurate predictions for GWs from $\mathrm{BBHs}$ should crucially also account for the BHs' spins [14], and this is an important aspect of the present work. The gravitational waveforms presented here are valid up to quadratic order in angular momenta (spins) of the compact stars; that is, we extend Crowley, Kovacs, and Thorne's seminal nonspinning result [15]. We also improve on our earlier reproduction of the nonspinning result [16] by presenting results in a compact Lorentz-covariant form, using an improved integration strategy.

To obtain these results we generalize the recently introduced worldline quantum field theory (WQFT) formalism $[16,17]$ to spinning particles on the worldline. This is achieved by including anticommuting worldline fields carrying the spin degrees of freedom, building upon Refs. [18-20]. Our formalism manifests an $\mathcal{N}=2$ extended worldline supersymmetry (SUSY) which holds up to the desired quadratic order in spin. The SUSY implies conservation of the covariant spin-supplementary condition (SSC), and thus represents an alternative formulation of the symmetries inherent to spinning bodies. It also operates on the spinning waveform.

The spinning WQFT innovates over previous approaches to classical spin based on corotating-frame variables $[21,22]$ in the effective field theory (EFT) of compact objects [23,24] — see Ref. [25] for the construction of PM integrands and Refs. [26,27] for worldline and spin deflections (in agreement with scattering amplitude results $[28,29])$. The worldline EFT was applied to radiation also in the weak-field and slow-motion, i.e., post-Newtonian, approximation [30]see Ref. [31] for more traditional methods. Other approaches to PM spin effects can be found in Ref. [32].

Spinning Worldline Quantum Field Theory. - It has been known since the 1980s [18] that the relativistic wave 
equation for a massless or massive spin- $\mathcal{N} / 2$ field in flat spacetime (generalizing the Klein-Gordon, Dirac, and Maxwell or Proca equations) may be obtained by quantization of an extended supersymmetric particle model where one augments the bosonic trajectory $x^{\mu}(\tau)$ by $\mathcal{N}$ anticommuting, real worldline fields. Generalizing this to a curved background spacetime comes with consistency problems beyond $\mathcal{N}=2$. Yet the situation for spins up to 1 is well understood [20], and sufficient for our purposes of describing two-body scattering up to quadratic order in spin.

We therefore augment the worldline trajectories $x_{i}^{\mu}\left(\tau_{i}\right)$ $(i=1,2)$ of our two massive bodies by anticommuting complex Grassmann fields $\psi_{i}^{a}\left(\tau_{i}\right)$. These are vectors in the flat tangent Minkowski spacetime connected to the curved spacetime via the vierbein $e_{\mu}^{a}(x)$. The worldline action in the massive case for each body takes the form (suppressing the $i$ subscripts) [33]

$S=-m \int d \tau\left[\frac{1}{2} g_{\mu \nu} \dot{x}^{\mu} \dot{x}^{\nu}+i \bar{\psi}_{a} \frac{D \psi^{a}}{D \tau}+\frac{1}{2} R_{a b c d} \bar{\psi}^{a} \psi^{b} \bar{\psi}^{c} \psi^{d}\right]$,

where $g_{\mu \nu}=e_{\mu}^{a} e_{\nu}^{b} \eta_{a b}$ is the metric in mostly minus signature, $\left(D \psi^{a} / D \tau\right)=\dot{\psi}^{a}+\dot{x}^{\mu} \omega_{\mu}{ }^{a}{ }_{b} \psi^{b}$ includes the spin connection $\omega_{\mu b}^{a}$, and the Riemann tensor is $R_{\mu \nu a b}=$ $e_{\mu}^{c} e_{\nu}^{d} R_{a b c d}=2\left(\partial_{[\mu} \omega_{\nu] a b}+\omega_{\left[\mu \mid a^{c}\right.} \omega_{\nu] c b}\right)$. This theory enjoys a global $\mathcal{N}=2$ SUSY: it is invariant under

$\delta x^{\mu}=i \bar{\epsilon} \psi^{\mu}+i \epsilon \bar{\psi}^{\mu}, \quad \delta \psi^{a}=-\epsilon e_{\mu}^{a} \dot{x}^{\mu}-\delta x^{\mu} \omega_{\mu b}^{a} \psi^{b}$,

with constant SUSY parameters $\epsilon$ and $\bar{\epsilon}=\epsilon^{\dagger}$.

The connection to a traditional description of spinning bodies in general relativity, using the spin field $S^{\mu \nu}$ and the Lorentz body-fixed frame $\Lambda_{\mu}^{A}[21,22,24,34,35]$, comes about upon identifying the spin field $S^{\mu \nu}(\tau)$ with the Grassmann bilinear:

$$
S^{\mu \nu}=-2 i e_{a}^{\mu} e_{b}^{\nu} \bar{\psi}^{[a} \psi^{b]} .
$$

One can easily show that $S^{a b}$ obeys the Lorentz algebra under Poisson brackets $\left\{\psi^{a}, \bar{\psi}^{b}\right\}_{\mathrm{PB}}=-i \eta^{a b}$. In fact, the spin-supplementary condition (SSC) and preservation of spin length may be related to $\mathcal{N}=2$ SUSY-related constraints [33]. Finally, by deriving the classical equations of motion from the action these can be shown to match the Mathisson-Papapetrou equations [36] at quadratic spin order. This indicates a hidden $\mathcal{N}=2$ SUSY in the actions of Refs. [22,34,35].

The actions of Refs. [22,34,35] also carry a first spininduced multipole moment term at quadratic order in spins with an undertermined Wilson coefficient $C_{E}$, where here $C_{E}=0$ for a Kerr BH. Translating it to our formalism this term reads

$$
S_{E S^{2}}:=-m \int d \tau C_{E} E_{a b} \bar{\psi}^{a} \psi^{b} \bar{\psi} \cdot \psi,
$$

where $E_{a b}:=R_{a \mu b \nu} \dot{x}^{\mu} \dot{x}^{\nu}$ is the "electric" part of the Riemann tensor. The $\mathcal{N}=2$ SUSY is now maintained only in an approximate sense [33]: it survives in the action for terms up to $\mathcal{O}\left(\psi^{5}\right)$, i.e., quadratic order in spin.

In order to describe a scattering scenario we expand the worldline fields about solutions of the equations of motion along straight-line trajectories:

$$
\begin{aligned}
x_{i}^{\mu}\left(\tau_{i}\right) & =b_{i}^{\mu}+v_{i}^{\mu} \tau_{i}+z_{i}^{\mu}\left(\tau_{i}\right), \\
\psi_{i}^{a}\left(\tau_{i}\right) & =\Psi_{i}^{a}+\psi_{i}^{\prime a}\left(\tau_{i}\right),
\end{aligned}
$$

where $\mathcal{S}_{i}^{\mu \nu}:=-2 i \bar{\Psi}_{i}^{[\mu} \Psi_{i}^{\nu]}$ captures the initial spin of the two massive objects. The weak gravity expansion of the vierbein reads

$$
e_{\mu}^{a}=\eta^{a \nu}\left(\eta_{\mu \nu}+\frac{\kappa}{2} h_{\mu \nu}-\frac{\kappa^{2}}{8} h_{\mu \rho} h_{\nu}^{\rho}+\mathcal{O}\left(\kappa^{3}\right)\right),
$$

introducing the graviton field $h_{\mu \nu}(x)$ and the gravitational coupling $\kappa^{2}=32 \pi G$. Note that in this perturbative framework the distinction between curved $\mu, \nu, \ldots$ and tangent $a, b, \ldots$ indices necessarily drops.

The spinning WQFT has the partition function

$$
\begin{aligned}
\mathcal{Z}_{\mathrm{WQFT}}: & =\operatorname{const} \times \int D\left[h_{\mu \nu}\right] e^{i\left(S_{\mathrm{EH}}+S_{\mathrm{gf}}\right)} \\
& \times \int \prod_{i=1}^{2} D\left[z_{i}^{\mu}\right] D\left[\psi_{i}^{\prime \mu}\right] \exp \left[i \sum_{i=1}^{2} S^{(i)}+S_{E S^{2}}^{(i)}\right],
\end{aligned}
$$

where $S_{\mathrm{EH}}$ is the Einstein-Hilbert action, and the gaugefixing term $S_{\mathrm{gf}}$ enforces de Donder gauge. The SUSY variations [Eq. (2)] leave an imprint on the free energy (or eikonal) $F_{\mathrm{WQFT}}\left(b_{i}, v_{i}, \mathcal{S}_{i}\right):=-i \log \mathcal{Z}_{\mathrm{WQFT}}$ : after integrating out the fluctuations $z^{\mu}$ and $\psi^{\prime \mu}$ in the path integral [Eq. (7)], the SUSY variations of the background trajectories [Eq. (5)] remain intact in an asymptotically flat space time. That is, the transformations

$$
\begin{aligned}
\delta b_{i}^{\mu}= & i \bar{\epsilon} \Psi_{i}^{\mu}+i \epsilon \bar{\Psi}_{i}^{\mu}, \quad \delta v_{i}^{\mu}=0, \quad \delta \Psi_{i}^{\mu}=-\epsilon v_{i}^{\mu} \\
& \Rightarrow \delta \mathcal{S}_{i}^{\mu \nu}=v_{i}^{\mu} \delta b_{i}^{\nu}-v_{i}^{\nu} \delta b_{i}^{\mu}
\end{aligned}
$$

are a symmetry of $F_{\mathrm{WQFT}}\left(b_{i}, v_{i}, \mathcal{S}_{i}\right)$ (only up to quadratic spin order when the Wilson coefficients $C_{E, i}$ are included). As we shall see, this is also a symmetry of the waveform. Using a suitable shift of the proper times $\tau_{i}$ we may choose $b \cdot v_{i}=0$, where $b^{\mu}=b_{2}^{\mu}-b_{1}^{\mu}$ is the relative impact parameter; by gauge fixing the SUSY transformations [Eq. (8)] we impose $v_{i, \mu} \mathcal{S}_{i}^{\mu \nu}=0$ (the covariant SSC).

Feynman rules.-As the Feynman rules for the EinsteinHilbert action are conventional we will not dwell on them; the only subtlety is our use of a retarded graviton propagator:

$$
\mu \nu \underset{k}{\mu \nu} \rho \sigma=i \frac{P_{\mu \nu ; \rho \sigma}}{\left(k^{0}+i \epsilon\right)^{2}-\mathbf{k}^{2}},
$$




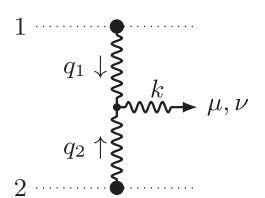

(a)

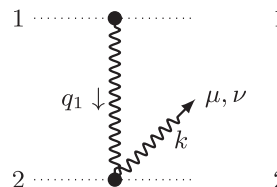

(c)

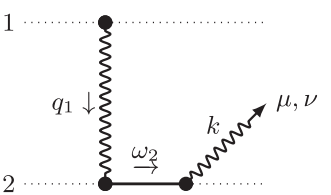

(b)

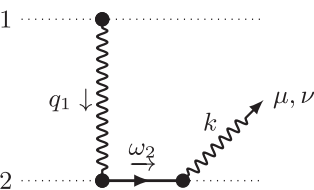

(d)
FIG. 1. The four diagram topologies contributing to the 2PM Bremsstrahlung up to $\mathcal{O}\left(\mathcal{S}^{2}\right)$, where $\omega_{i}=k \cdot v_{i}$ by energy conservation at the worldline vertices. For diagrams (b)-(d) we also include the corresponding flipped topologies with massive bodies $1 \leftrightarrow 2$; for diagram (d) (which includes the propagating fermion $\psi_{2}^{\prime \mu}$ ) we also include the graph with the arrow reversed.

with $P_{\mu \nu ; \rho \sigma}:=\eta_{\mu(\rho} \eta_{\sigma) \nu}-\frac{1}{2} \eta_{\mu \nu} \eta_{\rho \sigma}$. On the worldline we work in one-dimensional energy (frequency) space: the propagators for the fluctuations $z^{\mu}(\omega)$ and anticommuting vectors $\psi^{\prime \mu}(\omega)$ are respectively

$$
\begin{aligned}
& \stackrel{\mu}{\bullet} \underset{\omega}{\bullet} \cdots=-i \frac{\eta^{\mu \nu}}{m(\omega+i \epsilon)^{2}}, \\
& \underset{\omega}{\leftrightarrow} \stackrel{\nu}{\bullet}=-i \frac{\eta^{\mu \nu}}{m(\omega+i \epsilon)},
\end{aligned}
$$

which also both involve a retarded $i \epsilon$ prescription. The former was already used in Refs. [16,17].

Next we consider the worldline vertices. The simplest of these is the single-graviton emission vertex:

$$
\begin{aligned}
h_{\mu \nu}(k)= & -i \frac{m \kappa}{2} e^{i k \cdot b} \delta(k \cdot v)\left(v^{\mu} v^{\nu}+i k_{\rho} \mathcal{S}^{\rho(\mu} v^{\nu)}\right. \\
& \left.+\frac{1}{2} k_{\rho} k_{\sigma} \mathcal{S}^{\rho \mu} \mathcal{S}^{\nu \sigma}+\frac{C_{E}}{2} v^{\mu} v^{\nu}(k \cdot \mathcal{S} \cdot \mathcal{S} \cdot k)\right),
\end{aligned}
$$

where $\delta(\omega):=(2 \pi) \delta(\omega)$, and we have used $\mathcal{S}^{\mu \nu}=$ $-2 i \bar{\Psi}\left[\mu \Psi^{\nu l}\right.$. The other worldline-based vertices required for the 2PM Bremsstrahlung all appear in Fig. 1: the two-point interaction between a graviton and a single $z^{\mu}$ mode in (b), the two-graviton emission vertex in (c), and the two-point interaction between a graviton and $\psi^{\prime \mu}$ in (d). Full expressions for these vertices are provided in the Supplemental Material [37].

Waveform from WQFT.-To describe the Bremsstrahlung at $2 \mathrm{PM}$ order including spin effects we compute the expectation value $k^{2}\left\langle h_{\mu \nu}(k)\right\rangle_{\mathrm{WQFT}}$. This requires us to compute four kinds of Feynman graphs, illustrated in Fig. 1. Explicit expressions for the first two graphs (a) and (b) were given in the nonspinning case [16]; these are now modified by terms up to $\mathcal{O}\left(\mathcal{S}^{2}\right)$. Graphs (c) and (d) are unique to the spinning case-for the latter we sum over both routings of the fermion line.

From this result we seek to obtain the waveform in spacetime in the wave zone, where the distance to the observer $|\mathbf{x}|=r$ is large compared with all other lengths. Following Ref. [16] the gauge-invariant frequency-domain waveform $4 G \epsilon^{\mu \nu} S_{\mu \nu}\left[k^{\mu}=\Omega(1, \hat{\mathbf{x}})\right]$ is extracted from the WQFT via

$$
S_{\mu \nu}(k)=\frac{2}{\kappa} k^{2}\left\langle h_{\mu \nu}(k)\right\rangle_{\mathrm{WQFT}},
$$

where $\Omega$ is the GW frequency and $\hat{\mathbf{x}}=\mathbf{x} / r$ points towards the observer. However, it is advantageous to study the timedomain waveform $f(u, \hat{\mathbf{x}})$ which is given by a Fourier transform:

$\kappa \epsilon^{\mu \nu} h_{\mu \nu}=\frac{f(u, \hat{\mathbf{x}})}{r}=\left.\frac{4 G}{r} \int_{\Omega} e^{-i k \cdot x} \epsilon^{\mu \nu} S_{\mu \nu}(k)\right|_{k^{\mu}=\Omega \rho^{\mu}}$.

We have contracted with a polarization tensor $\epsilon^{\mu \nu}=\frac{1}{2} \epsilon^{\mu} \epsilon^{\nu}$, $\int_{\Omega}:=\int_{-\infty}^{\infty}(d \Omega / 2 \pi)$, and $\rho^{\mu}=(1, \hat{\mathbf{x}})$; in a PM decomposition $f=\sum_{n} G^{n} f^{(n)}$ we seek the 2PM component $f^{(2)}$. Note that $k \cdot x=\Omega(t-r)$ yields the retarded time $u=t-r$, and $\epsilon \cdot \epsilon=\epsilon \cdot \rho=0$.

Integration.-Our integration procedure follows closely that used for the nonspinning calculation in Ref. [16], the main difference being that we maintain four-dimensional Lorentz covariance. Each diagram contributing to $k^{2}\left\langle h_{\mu \nu}(k)\right\rangle_{\mathrm{WQFT}}$ carries the overall factor

$\mu_{1,2}(k)=e^{i\left(q_{1} \cdot b_{1}+q_{2} \cdot b_{2}\right)} \delta\left(q_{1} \cdot v_{1}\right) \delta\left(q_{2} \cdot v_{2}\right) \delta\left(k-q_{1}-q_{2}\right)$.

We integrate over $q_{i}$, the momentum emitted from each worldline (see Fig. 1). When we also integrate over $\Omega$-as in Eq. (13) — the full integration measure becomes

$$
\int_{\Omega, q_{1}, q_{2}} \mu_{1,2}(k) e^{-i k \cdot x}=\frac{1}{\rho \cdot v_{2}} \int_{q_{1}} \delta\left(q_{1} \cdot v_{1}\right) e^{-i q_{1} \cdot \tilde{b}},
$$

where $\int_{q_{i}}:=\int\left[d^{4} q_{i} /(2 \pi)^{4}\right]$; the delta function constraints give $\Omega=\left[\left(q_{1} \cdot v_{2}\right) /\left(\rho \cdot v_{2}\right)\right]$ and $q_{2}=k-q_{1}$. The shifted impact parameter,

$$
\tilde{b}^{\mu}=\tilde{b}_{2}^{\mu}-\tilde{b}_{1}^{\mu}, \quad \tilde{b}_{i}^{\mu}=b_{i}^{\mu}+u_{i} v_{i}^{\mu},
$$

extends the original impact parameter $b^{\mu}=b_{2}^{\mu}-b_{1}^{\mu}$ along the undeflected trajectories of the two bodies. Finally, $u_{i}$ is the retarded time in the $i$ th rest frame: 


$$
u_{i}=\frac{\rho \cdot\left(x-b_{i}\right)}{\rho \cdot v_{i}} \text {. }
$$

This implies $\rho \cdot \tilde{b}_{i}=\rho \cdot x=u$, so $\rho \cdot \tilde{b}=0$.

Rewriting the integral measure as in Eq. (15) is convenient for performing the integrals of diagrams (b)-(d) of Fig. 1, in the rest frame of body 1. The mirrored counterparts to these diagrams are easily recovered after integration using the $1 \leftrightarrow 2$ symmetry of the waveform. To integrate diagram (a) we insert the partial-fraction identity $q_{1}^{-2} q_{2}^{-2}=-q_{1}^{-2}\left(2 k \cdot q_{1}\right)^{-1}-q_{2}^{-2}\left(2 k \cdot q_{2}\right)^{-1}$ (which is valid for $k$ on shell) and focus on the first term.

The full 2PM waveform is then written schematically as (dropping the subscript on $q_{1}$ )

$$
\begin{aligned}
\frac{f^{(2)}}{m_{1} m_{2}}= & 4 \pi \int_{q} \delta\left(q \cdot v_{1}\right) \frac{e^{-i q \cdot \tilde{b}}}{q^{2}}\left(\frac{\mathcal{N}(q)}{q \cdot v_{2}+i \epsilon}+\frac{\mathcal{M}(q)}{\left(q \cdot v_{2}\right)(q \cdot \rho)}\right), \\
& +(1 \leftrightarrow 2),
\end{aligned}
$$

with the $\mathcal{N}$ and $\mathcal{M}$ contributions corresponding to diagrams (b)-(d) and (a) in Fig. 1 respectively. The numerators $\mathcal{N}(q)$ and $\mathcal{M}(q)$ have a uniform power counting in $q$ for each spin order,

$$
\begin{aligned}
& \mathcal{N}(q)=\mathcal{N}_{\mu} q^{\mu}+\mathcal{N}_{\mu \nu} q^{\mu} q^{\nu}+\mathcal{N}_{\mu \nu \rho} q^{\mu} q^{\nu} q^{\rho}, \\
& \mathcal{M}(q)=\mathcal{M}_{\mu \nu} q^{\mu} q^{\nu}+\mathcal{M}_{\mu \nu \rho} q^{\mu} q^{\nu} q^{\rho}+\mathcal{M}_{\mu \nu \rho \sigma} q^{\mu} q^{\nu} q^{\rho} q^{\sigma},
\end{aligned}
$$

and the nonspinning result involves only $\mathcal{N}_{\mu}$ and $\mathcal{M}_{\mu \nu}$. We present full expressions for $\mathcal{N}$ and $\mathcal{M}$ in the ancillary file attached to the arXiv submission of this Letter.

To the lowest order in $q^{\mu}$, the first integral in Eq. (18) is

$$
\begin{aligned}
4 \pi & \int_{q} \delta\left(q \cdot v_{1}\right) \frac{e^{-i q \cdot \tilde{b}}}{q^{2}} \frac{q^{\mu}}{q \cdot v_{2}+i \epsilon} \\
& =\frac{P_{1}^{\mu \nu} v_{2, \nu}}{\left(\gamma^{2}-1\right)|\tilde{\mathbf{b}}|_{1}}-\frac{b^{\mu}}{|b|^{2}}\left(\frac{1}{\sqrt{\gamma^{2}-1}}+\frac{u_{2}}{|\tilde{\mathbf{b}}|_{1}}\right),
\end{aligned}
$$

where $P_{i}^{\mu \nu}:=\eta^{\mu \nu}-v_{i}^{\mu} v_{i}^{\nu}$ is a projector into the rest frame of the $i$ th body, $|b|=-\sqrt{b^{\mu} b_{\mu}}$ (the impact parameter is spacelike) and

$$
|\tilde{\mathbf{b}}|_{1,2}:=\sqrt{-\tilde{b}_{\mu} P_{1,2}^{\mu \nu} \tilde{b}_{\nu}}=\sqrt{|b|^{2}+\left(\gamma^{2}-1\right) u_{2,1}^{2}}
$$

are the lengths of the shifted impact parameter $\tilde{b}^{\mu}$ [Eq. (16)] in the two rest frames. The second integral in Eq. (18) is

$$
\begin{aligned}
4 \pi & \int_{q} \delta\left(q \cdot v_{1}\right) \frac{e^{-i q \cdot \tilde{b}}}{q^{2}} \frac{q^{\mu} q^{\nu}}{q \cdot v_{2} q \cdot \rho} \\
& =\frac{K_{1}^{\mu \nu} v_{2} \cdot K_{1} \cdot \rho-2\left(v_{2} \cdot K_{1}\right)^{(\mu}\left(\rho \cdot K_{1}\right)^{\nu)}}{\left(\gamma^{2}-1\right)\left(\rho \cdot v_{1}\right)^{2}|b|^{2}|\tilde{b}|^{2}|\tilde{\mathbf{b}}|_{1}},
\end{aligned}
$$

where we have introduced the symmetric tensor

$$
K_{i}^{\mu \nu}:=P_{i}^{\mu \nu}|\tilde{\mathbf{b}}|_{i}^{2}+\left(P_{i} \cdot \tilde{b}\right)^{\mu}\left(P_{i} \cdot \tilde{b}\right)^{\nu},
$$

with the property that $K_{i}^{\mu \nu} v_{i, \nu}=K_{i}^{\mu \nu} \tilde{b}_{\nu}=0$. Both integrals are derived in the Supplemental Material [37]; one generalizes to higher powers of $q^{\mu}$ in the numerators by taking derivatives with respect to $\tilde{b}^{\mu}$.

Results.-The 2PM waveform takes the schematic form

$$
\frac{f^{(2)}}{m_{1} m_{2}}=\sum_{s=0}^{2} \frac{1}{|\tilde{\mathbf{b}}|_{1}^{2 s+1}}\left[\alpha_{1}^{(s)}+\frac{\beta_{1}^{(s)}}{|\tilde{b}|^{2 s+2}}\right]+(1 \leftrightarrow 2),
$$

where the coefficients $\alpha_{i}^{(s)}, \beta_{i}^{(s)}$, provided in the ancillary file, are associated with the $\mathcal{N}$ - and $\mathcal{M}$-type contributions in Eq. (18) respectively; they are functions of $u_{i}, b^{\mu}, v_{i}^{\mu}, \rho^{\mu}$, and $\mathcal{S}_{i}^{\mu \nu}$ and bilinear in $\epsilon^{\mu}$. The waveform $f$ is invariant under the SUSY transformations in Eq. (8) to quadratic order in spin regardless of the values of $C_{E, i}$. To see this we expand the waveform at all PM orders in powers of spin:

$f=f_{0}+\sum_{i=1}^{2} \mathcal{S}_{i, \mu \nu} f_{i}^{\mu \nu}+\sum_{i, j=1}^{2} \mathcal{S}_{i, \mu \nu} \mathcal{S}_{j, \rho \sigma} f_{i j}^{\mu \nu ; \rho \sigma}+\mathcal{O}\left(\mathcal{S}^{3}\right)$,

where $f_{i}^{\mu \nu}$ and $f_{i j}^{\mu \nu ; \rho \sigma}$ are defined modulo terms that vanish on support of $v_{i, \mu} \mathcal{S}_{i}^{\mu \nu}=0$. The SUSY links higher-spin to lower-spin terms:

$$
\frac{1}{2} \frac{\partial f_{0}}{\partial b_{i, \mu}}=v_{i, \nu} f_{i}^{[\mu \nu]}, \quad \frac{1}{4} \frac{\partial f_{i}^{\mu \nu}}{\partial b_{j, \rho}}=v_{j, \sigma} f_{i j}^{\mu \nu ;[\rho \sigma]},
$$

and these identities are satisfied by the waveform [Eq. (24)].

To illustrate the waveform we consider the gravitational wave memory $\Delta f(\hat{\mathbf{x}}):=f(+\infty, \hat{\mathbf{x}})-f(-\infty, \hat{\mathbf{x}})$. The constant spin tensors are decomposed in terms of the PauliLubanski vectors $a_{i}^{\mu}$ as $\mathcal{S}_{i}^{\mu \nu}=\epsilon_{\rho \sigma}^{\mu \nu} v_{i}^{\rho} a_{i}^{\sigma}$, the latter satisfying $a_{i} \cdot v_{i}=0$. In the aligned-spin case $a_{i} \cdot b=a_{i} \cdot v_{j}=0$, i.e., the spin vectors are orthogonal to the plane of scattering. Writing $\left|a_{i}\right|=\sqrt{-a_{i}^{2}}$ the wave memory is then proportional to the nonspinning result:

$$
\begin{aligned}
\Delta f^{(2)}= & \left(1+\frac{2 v\left|a_{3}\right|}{b\left(1+v^{2}\right)}+\frac{\left|a_{3}\right|^{2}}{|b|^{2}}-\sum_{i=1}^{2} \frac{C_{E, i}\left|a_{i}\right|^{2}}{|b|^{2}}\right) \Delta f_{\mathcal{S}=0}^{(2)} \\
\frac{\Delta f_{\mathcal{S}=0}^{(2)}}{m_{1} m_{2}}= & \frac{4\left(2 \gamma^{2}-1\right) \epsilon \cdot v_{1}\left(2 b \cdot \epsilon \rho \cdot v_{1}-b \cdot \rho \epsilon \cdot v_{1}\right)}{|b|^{2} \sqrt{\gamma^{2}-1}\left(\rho \cdot v_{1}\right)^{2}} \\
& +(1 \leftrightarrow 2),
\end{aligned}
$$

where $a_{3}^{\mu}=a_{1}^{\mu}+a_{2}^{\mu}$. For two Kerr black holes $\left(C_{E, i}=0\right)$ with equal-and-opposite spins $\left(a_{1}^{\mu}=-a_{2}^{\mu}\right)$ we see that $\Delta f^{(2)}=\Delta f_{\mathcal{S}=0}^{(2)}$, which we observe also when the spins are misaligned to the plane of scattering.

There is also a 1PM (nonradiating) contribution to the waveform consisting of single-graviton emission from either massive body:

$$
f^{(1)}(\hat{\mathbf{x}})=\frac{2 m_{1}}{\rho \cdot v_{1}}\left(\epsilon \cdot v_{1}\right)^{2}+\frac{2 m_{2}}{\rho \cdot v_{2}}\left(\epsilon \cdot v_{2}\right)^{2} .
$$




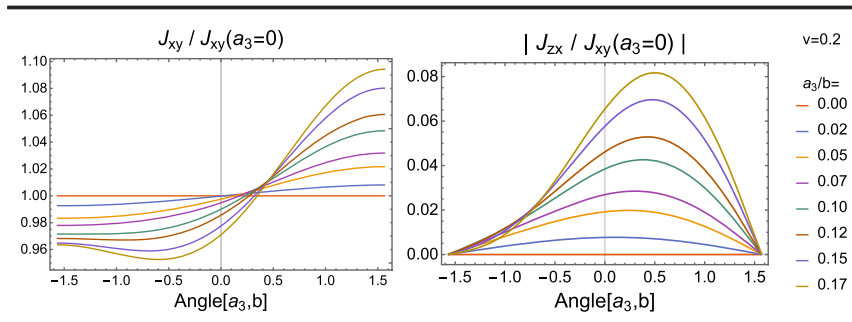

FIG. 2. Total radiated angular momenta for the scattering of two Kerr-BHs with $v=0.2$ as a function of the angle between the total initial spins $\mathbf{a}_{3}=\mathbf{a}_{1}+\mathbf{a}_{2}$ and $\mathbf{b}$ (with $a_{i} \cdot \mathbf{v}_{i}=0$ ) for a range of ratios $\left|\mathbf{a}_{3}\right| /|\mathbf{b}|$. We show the normalized ratio of angular momenta emitted orthogonal to the $\mathbf{b}, \mathbf{v}$ plane (left plot) and in the b direction (right plot), normalization is w.r.t. angular momentum emitted in the spinless case.

At 1PM order there is manifestly no dependence on either the spins $\mathcal{S}_{i}^{\mu \nu}$ or impact parameters $b_{i}^{\mu}$, so the SUSY identities in Eq. (26) are trivially satisfied.

Finally, the wave memory and 1PM part of the waveform contribute to the total radiated angular momentum $J_{i j}^{\mathrm{rad}}$. Using three-dimensional Cartesian basis vectors $\hat{\mathbf{e}}_{i}$, we choose a frame of reference with the initial velocities $v_{i}^{\mu}$ restricted to the $t-x$ plane; $\mathbf{b}=|b| \hat{\mathbf{e}}_{2}$ is orthogonal to these. Then we find two nonzero components of $J_{i j}^{\mathrm{rad}}: J_{x y}^{\mathrm{rad}}$ and $J_{z x}^{\mathrm{rad}}$, which are conveniently arranged into

$$
\begin{aligned}
\frac{J_{x y}^{\mathrm{rad}}+i J_{z x}^{\mathrm{rad}}}{\left.J_{x y}^{\mathrm{init}}\right|_{\mathcal{S}=0}}= & \frac{4 G^{2} m_{1} m_{2}}{|b|^{2}} \frac{\left(2 \gamma^{2}-1\right)}{\sqrt{\gamma^{2}-1}} \mathcal{I}(v) \\
& \times\left(1-\frac{2 i v \mathbf{a}_{3} \cdot \mathbf{l}}{|b|\left(1+v^{2}\right)}-\frac{\left(\mathbf{a}_{3} \cdot \mathbf{l}\right)^{2}}{|b|^{2}}\right. \\
& \left.+\sum_{i=1}^{2} \frac{C_{E, i}}{|b|^{2}}\left(\mathbf{a}_{i} \cdot \mathbf{l}\right)^{2}\right)+\mathcal{O}\left(G^{3}\right) .
\end{aligned}
$$

We normalize with respect to $\left.J_{x y}^{\text {init }}\right|_{\mathcal{S}=0}$, the initial angular momentum in the nonspinning case. The spin vectors $\mathbf{a}_{1}$ and $\mathbf{a}_{2}$ are taken in the rest frame of each massive body; $\mathbf{a}_{3}=\mathbf{a}_{1}+\mathbf{a}_{2}, \mathbf{l}=\hat{\mathbf{e}}_{2}+i \hat{\mathbf{e}}_{3}$, and

$$
\mathcal{I}(v)=-\frac{8}{3}+\frac{1}{v^{2}}+\frac{\left(3 v^{2}-1\right)}{v^{3}} \operatorname{arctanh}(v)
$$

is a universal prefactor. Equation (29) holds in the rest frame of either body or the center-of-mass (c.o.m.) frame; see Fig. 2 for plots. For a derivation we refer the reader to the Supplemental Material [37]. There we also compute the total radiated energy in the c.o.m. frame. Due to the multiscale nature of the waveform it is difficult to perform the necessary time and solid angle integrals, so we performed a low velocity expansion. For terms up to $\mathcal{O}\left(v^{2}\right)$ we find

$$
\begin{aligned}
E_{\mathrm{com}}^{\mathrm{rad}, \mathrm{LO}}= & \frac{v G^{3} m_{1}^{2} m_{2}^{2} \pi}{|b|^{3}}\left[\frac{37}{15}+\frac{v\left(65 m_{1}+69 m_{2}\right)\left(\mathbf{a}_{1} \cdot \hat{\mathbf{e}}_{3}\right)}{10|b|\left(m_{1}+m_{2}\right)}+\frac{1503\left(\mathbf{a}_{1} \cdot \hat{\mathbf{e}}_{1}\right)\left(\mathbf{a}_{2} \cdot \hat{\mathbf{e}}_{1}\right)-3559\left(\mathbf{a}_{1} \cdot \hat{\mathbf{e}}_{2}\right)\left(\mathbf{a}_{2} \cdot \hat{\mathbf{e}}_{2}\right)+1816\left(\mathbf{a}_{1} \cdot \hat{e}_{3}\right)\left(\mathbf{a}_{2} \cdot \hat{\mathbf{e}}_{3}\right)}{320|b|^{2}}\right. \\
& \left.+\frac{9\left(185-176 C_{E, 1}\right)\left(\mathbf{a}_{1} \cdot \hat{\mathbf{e}}_{1}\right)^{2}-\left(3385-3472 C_{E, 1}\right)\left(\mathbf{a}_{1} \cdot \hat{e}_{2}\right)^{2}+8\left(245-236 C_{E, 1}\right)\left(\mathbf{a}_{1} \cdot \hat{\mathbf{e}}_{3}\right)^{2}}{320|b|^{2}}+(1 \leftrightarrow 2)+\mathcal{O}\left(v^{2}\right)\right], \quad(31)
\end{aligned}
$$

where the swap $(1 \leftrightarrow 2)$ does not affect the basis vectors $\hat{\mathbf{e}}_{i}$ or the constant term $(37 / 15)$. It is straightforward to extend this result to higher orders in $v$.

Conclusions. - In this Letter we extended the WQFT to describe spinning compact bodies to quadratic order in spin, and calculated the leading-PM order waveform for highly eccentric (scattering) orbits. Our accompanying work [33] presents an application to further observables such as the spin kick and deflection [26,29] at 2PM order and gives details on the approximate SUSY and its relation to the SSC. The radiated energy [Eq. (31)] should also be particularly useful for future studies. In Refs. [38,39] the $\mathcal{O}\left(G^{3}\right)$ energy loss from a scattering of nonspinning black holes was recently computed to all orders in velocity using the formalism of [40] (see also Ref. [41]); a similar result could conceivably be obtained at $\mathcal{O}\left(\mathcal{S}^{2}\right)$, and then checked against Eq. (31) in the low-velocity limit. Similarly, the remarkably simple result for radiated angular momentum [Eq. (29)] at 2PM order is intriguing; it may be important for understanding the high-energy limit; see Ref. [42,43] for the nonspinning case.
The application of modern on shell and integration techniques to compute scattering amplitudes $[38,44-48]$ holds great promise for pushing calculations to higher PM orders. This is demonstrated by the impressive calculation of the 4PM conservative dynamics in the potential region [48,49] - see also Refs. [42,43,46,50-54]. The connection between amplitudes and classical physics was studied in Refs. [40,41,55], and Ref. [27] discussed the connection to bound orbits. Our WQFT framework $[16,17]$ provides an efficient, rather intuitive way to connect amplitude and (classical) worldline EFT calculations. It may therefore benefit from modern amplitude techniques at higher PM orders in future work, building on the compact Lorentzcovariant master integrals provided here.

We would like to thank F. Bautista, R. Bonezzi, A. Buonanno, P. Pichini, and J. Vines for very helpful discussions. We are also grateful for use of G. Kälin's $\mathrm{C}++$ graph library. G. U. J.'s and G. M.'s research is funded by the Deutsche Forschungsgemeinschaft (DFG, German Research Foundation) Project No. 417533893/GRK2575 "Rethinking Quantum Field Theory." 
*gustav.uhre.jakobsen@physik.hu-berlin.de

gustav.mogull@aei.mpg.de

*jan.plefka@hu-berlin.de

\$an.steinhoff@aei.mpg.de

[1] B. P. Abbott et al. (LIGO Scientific, Virgo Collaborations), Observation of Gravitational Waves from a Binary Black Hole Merger, Phys. Rev. Lett. 116, 061102 (2016); GWTC-1: A Gravitational-Wave Transient Catalog of Compact Binary Mergers Observed by LIGO and Virgo during the First and Second Observing Runs, Phys. Rev. X 9, 031040 (2019); GWTC-2: Compact binary coalescences observed by LIGO and Virgo during the first half of the third observing run, arXiv:2010.14527.

[2] R. Abbott et al. (LIGO Scientific, Virgo Collaborations), Population properties of compact objects from the second LIGO-Virgo gravitational-wave transient catalog, Astrophys. J. Lett. 913, L7 (2021).

[3] R. Abbott et al. (LIGO Scientific, Virgo Collaborations), Tests of general relativity with binary black holes from the second LIGO-Virgo gravitational-wave transient catalog, Phys. Rev. D 103, 122002 (2021).

[4] B. P. Abbott et al. (LIGO Scientific, Virgo Collaborations), A gravitational-wave measurement of the hubble constant following the second observing run of Advanced LIGO and Virgo, Astrophys. J. 909, 218 (2021).

[5] B. P. Abbott et al. (LIGO Scientific, Virgo Collaborations), GW170817: Measurements of Neutron Star Radii and Equation of State, Phys. Rev. Lett. 121, 161101 (2018).

[6] J. Aasi et al. (LIGO Scientific Collaboration), Advanced LIGO, Classical Quantum Gravity 32, 115012 (2015); F. Acernese et al. (VIRGO Collaboration), Advanced Virgo: A second-generation interferometric gravitational wave detector, Classical Quantum Gravity 32, 024001 (2015); Y. Aso, Y. Michimura, K. Somiya, M. Ando, O. Miyakawa, T. Sekiguchi, D. Tatsumi, and H. Yamamoto (KAGRA Collaboration), Interferometer design of the KAGRA gravitational wave detector, Phys. Rev. D 88, 043007 (2013).

[7] M. Pürrer and C.-J. Haster, Gravitational waveform accuracy requirements for future ground-based detectors, Phys. Rev. Research 2, 023151 (2020).

[8] A. Buonanno and T. Damour, Effective one-body approach to general relativistic two-body dynamics, Phys. Rev. D 59, 084006 (1999); P. Ajith et al., Phenomenological template family for black-hole coalescence waveforms, Classical Quantum Gravity 24, S689 (2007).

[9] M. van de Meent and H. P. Pfeiffer, Intermediate Mass-Ratio Black Hole Binaries: Applicability of Small Mass-Ratio Perturbation Theory, Phys. Rev. Lett. 125, 181101 (2020).

[10] A. Ramos-Buades, S. Husa, G. Pratten, H. Estellés, C. García-Quirós, M. Mateu-Lucena, M. Colleoni, and R. Jaume, First survey of spinning eccentric black hole mergers: Numerical relativity simulations, hybrid waveforms, and parameter estimation, Phys. Rev. D 101, 083015 (2020); D. Chiaramello and A. Nagar, Faithful analytical effective-one-body waveform model for spin-aligned, moderately eccentric, coalescing black hole binaries, Phys. Rev. D 101, 101501 (2020); A. Nagar, A. Bonino, and P. Rettegno, Effective one-body multipolar waveform model for spin-aligned, quasicircular, eccentric, hyperbolic black hole binaries, Phys. Rev. D 103, 104021 (2021); X. Liu,
Z. Cao, and Z.-H. Zhu, A higher-multipole gravitational waveform model for an eccentric binary black holes based on the effective-one-body-numerical-relativity formalism, arXiv:2102.08614; M. Khalil, A. Buonanno, J. Steinhoff, and J. Vines, Radiation-reaction force and multipolar waveforms for eccentric, spin-aligned binaries in the effectiveone-body formalism, arXiv:2104.11705; T. Hinderer and S. Babak, Foundations of an effective-one-body model for coalescing binaries on eccentric orbits, Phys. Rev. D 96, 104048 (2017); T. Islam, V. Varma, J. Lodman, S. E. Field, G. Khanna, M. A. Scheel, H. P. Pfeiffer, D. Gerosa, and L. E. Kidder, Eccentric binary black hole surrogate models for the gravitational waveform and remnant properties: Comparable mass, nonspinning case, Phys. Rev. D 103, 064022 (2021).

[11] J. Samsing, Eccentric black hole mergers forming in globular clusters, Phys. Rev. D 97, 103014 (2018); C. L. Rodriguez, P. Amaro-Seoane, S. Chatterjee, and F. A. Rasio, Post-Newtonian Dynamics in Dense Star Clusters: HighlyEccentric, Highly-Spinning, and Repeated Binary Black Hole Mergers, Phys. Rev. Lett. 120, 151101 (2018); L. Gondán and B. Kocsis, High eccentricities and high masses characterize gravitational-wave captures in galactic nuclei as seen by earth-based detectors, arXiv:2011.02507.

[12] B. P. Abbott et al. (LIGO Scientific, Virgo Collaborations), Binary black hole population properties inferred from the first and second observing runs of Advanced LIGO and Advanced Virgo, Astrophys. J. Lett. 882, L24 (2019).

[13] B. Kocsis, M. E. Gaspar, and S. Marka, Detection rate estimates of gravity-waves emitted during parabolic encounters of stellar black holes in globular clusters, Astrophys. J. 648, 411 (2006); S. Mukherjee, S. Mitra, and S. Chatterjee, Detectability of hyperbolic encounters of compact stars with ground-based gravitational waves detectors, Mon. Not. R. Astron. Soc. 508, 5064 (2021); M. Zevin, J. Samsing, C. Rodriguez, C.-J. Haster, and E. Ramirez-Ruiz, Eccentric black hole mergers in dense star clusters: The role of binary-binary encounters, Astrophys. J. 871, 91 (2019); R. Gamba, M. Breschi, G. Carullo, P. Rettegno, S. Albanesi, S. Bernuzzi, and A. Nagar, GW190521: A dynamical capture of two black holes, arXiv:2106.05575.

[14] B. Zackay, T. Venumadhav, L. Dai, J. Roulet, and M. Zaldarriaga, Highly spinning and aligned binary black hole merger in the Advanced LIGO first observing run, Phys. Rev. D 100, 023007 (2019); Y. Huang, C.-J. Haster, S. Vitale, A. Zimmerman, J. Roulet, T. Venumadhav, B. Zackay, L. Dai, and M. Zaldarriaga, Source properties of the lowest signal-to-noise-ratio binary black hole detections, Phys. Rev. D 102, 103024 (2020).

[15] K. S. Thorne and S. J. Kovacs, The generation of gravitational waves. I. Weak-field sources, Astrophys. J. 200, 245 (1975); R. J. Crowley and K. S. Thorne, The generation of gravitational waves. II. The postlinear formation revisited, Astrophys. J. 215, 624 (1977); S. J. Kovacs and K. S. Thorne, The generation of gravitational waves. 3. Derivation of bremsstrahlung formulas, Astrophys. J. 217, 252 (1977); The generation of gravitational waves. 4. Bremsstrahlung, Astrophys. J. 224, 62 (1978).

[16] G. Uhre Jakobsen, G. Mogull, J. Plefka, and J. Steinhoff, Classical Gravitational Bremsstrahlung from a Worldline 
Quantum Field Theory, Phys. Rev. Lett. 126, 201103 (2021).

[17] G. Mogull, J. Plefka, and J. Steinhoff, Classical black hole scattering from a worldline quantum field theory, J. High Energy Phys. 02 (2021) 048.

[18] P. S. Howe, S. Penati, M. Pernici, and P. K. Townsend, Wave equations for arbitrary spin from quantization of the extended supersymmetric spinning particle, Phys. Lett. B 215, 555 (1988).

[19] G. W. Gibbons, R. H. Rietdijk, and J. W. van Holten, SUSY in the sky, Nucl. Phys. B404, 42 (1993).

[20] F. Bastianelli, P. Benincasa, and S. Giombi, Worldline approach to vector and antisymmetric tensor fields, J. High Energy Phys. 04 (2005) 010; Worldline approach to vector and antisymmetric tensor fields. II., J. High Energy Phys. 10 (2005) 114.

[21] R. A. Porto, Post-Newtonian corrections to the motion of spinning bodies in NRGR, Phys. Rev. D 73, 104031 (2006).

[22] M. Levi and J. Steinhoff, Spinning gravitating objects in the effective field theory in the post-Newtonian scheme, J. High Energy Phys. 09 (2015) 219.

[23] W. D. Goldberger and I. Z. Rothstein, An effective field theory of gravity for extended objects, Phys. Rev. D 73, 104029 (2006); Towers of gravitational theories, Gen. Relativ. Gravit. 38, 1537 (2006); W. D. Goldberger and A. Ross, Gravitational radiative corrections from effective field theory, Phys. Rev. D 81, 124015 (2010).

[24] R. A. Porto, The effective field theorist's approach to gravitational dynamics, Phys. Rep. 633, 1 (2016); M. Levi, Effective field theories of post-Newtonian gravity: A comprehensive review, Rep. Prog. Phys. 83, 075901 (2020).

[25] W. D. Goldberger, J. Li, and S. G. Prabhu, Spinning particles, axion radiation, and the classical double copy, Phys. Rev. D 97, 105018 (2018); W. D. Goldberger and A. K. Ridgway, Radiation and the classical double copy for color charges, Phys. Rev. D 95, 125010 (2017); C.-H. Shen, Gravitational radiation from color-kinematics duality, J. High Energy Phys. 11 (2018) 162.

[26] Z. Liu, R. A. Porto, and Z. Yang, Spin effects in the effective field theory approach to post-Minkowskian conservative dynamics, J. High Energy Phys. 06 (2021) 012.

[27] G. Kälin and R. A. Porto, Post-Minkowskian effective field theory for conservative binary dynamics, J. High Energy Phys. 11 (2020) 106.

[28] Z. Bern, A. Luna, R. Roiban, C.-H. Shen, and M. Zeng, Spinning black hole binary dynamics, scattering amplitudes and effective field theory, Phys. Rev. D 104, 065014 (2021).

[29] D. Kosmopoulos and A. Luna, Quadratic-in-spin Hamiltonian at $\mathcal{O}\left(\mathrm{G}^{2}\right)$ from scattering amplitudes, J. High Energy Phys. 07 (2021) 037.

[30] R. A. Porto, A. Ross, and I. Z. Rothstein, Spin induced multipole moments for the gravitational wave flux from binary inspirals to third Post-Newtonian order, J. Cosmol. Astropart. Phys. 03 (2011) 009; Spin induced multipole moments for the gravitational wave amplitude from binary inspirals to 2.5 Post-Newtonian order, J. Cosmol. Astropart. Phys. 09 (2012) 028; N. T. Maia, C. R. Galley, A. K. Leibovich, and R. A. Porto, Radiation reaction for spinning bodies in effective field theory I: Spin-orbit effects, Phys. Rev. D 96, 084064 (2017); Radiation reaction for spinning bodies in effective field theory II: Spin-spin effects, Phys. Rev. D 96, 084065 (2017); G. Cho, B. Pardo, and R. A. Porto, Gravitational radiation from inspiralling compact objects: Spin-spin effects completed at the next-to-leading post-Newtonian order, arXiv:2103.14612.

[31] C. Kant Mishra, A. Kela, K. G. Arun, and G. Faye, Readyto-use post-Newtonian gravitational waveforms for binary black holes with nonprecessing spins: An update, Phys. Rev. D 93, 084054 (2016); A. Buonanno, G. Faye, and T. Hinderer, Spin effects on gravitational waves from inspiraling compact binaries at second post-Newtonian order, Phys. Rev. D 87, 044009 (2013).

[32] J. Vines, Scattering of two spinning black holes in postMinkowskian gravity, to all orders in spin, and effective-onebody mappings, Classical Quantum Gravity 35, 084002 (2018); D. Bini and T. Damour, Gravitational spin-orbit coupling in binary systems, post-Minkowskian approximation and effective one-body theory, Phys. Rev. D 96, 104038 (2017); Gravitational spin-orbit coupling in binary systems at the second post-Minkowskian approximation, Phys. Rev. D 98, 044036 (2018); A. Guevara, Holomorphic classical limit for spin effects in gravitational and electromagnetic scattering, J. High Energy Phys. 04 (2019) 033; J. Vines, J. Steinhoff, and A. Buonanno, Spinning-black-hole scattering and the test-black-hole limit at second post-Minkowskian order, Phys. Rev. D 99, 064054 (2019); A. Guevara, A. Ochirov, and J. Vines, Scattering of spinning black holes from exponentiated soft factors, J. High Energy Phys. 09 (2019) 056; M.-Z. Chung, Y.-T. Huang, J.-W. Kim, and S. Lee, The simplest massive S-matrix: from minimal coupling to Black Holes, J. High Energy Phys. 04 (2019) 156; A. Guevara, A. Ochirov, and J. Vines, Black-hole scattering with general spin directions from minimal-coupling amplitudes, Phys. Rev. D 100, 104024 (2019); M.-Z. Chung, Y.-T. Huang, and J.-W. Kim, Classical potential for general spinning bodies, J. High Energy Phys. 09 (2020) 074; P. H. Damgaard, K. Haddad, and A. Helset, Heavy black hole effective theory, J. High Energy Phys. 11 (2019) 070; R. Aoude, K. Haddad, and A. Helset, On-shell heavy particle effective theories, J. High Energy Phys. 05 (2020) 051; A. Guevara, B. Maybee, A. Ochirov, D. O'Connell, and J. Vines, A worldsheet for Kerr, arXiv:2012.11570.

[33] G. Uhre Jakobsen, G. Mogull, J. Plefka, and J. Steinhoff, SUSY in the Sky with Gravitons, arXiv:2109.04465.

[34] J. Vines, D. Kunst, J. Steinhoff, and T. Hinderer, Canonical Hamiltonian for an extended test body in curved spacetime: To quadratic order in spin, Phys. Rev. D 93, 103008 (2016).

[35] R. A. Porto and I. Z. Rothstein, Next to leading order Spin(1) Spin(1) effects in the motion of inspiralling compact binaries, Phys. Rev. D 78, 044013 (2008); 81, 029905(E) (2010).

[36] M. Mathisson, Neue mechanik materieller systeme, Acta Phys. Pol. 6, 163 (1937).A. Papapetrou, Spinning test particles in general relativity. 1, Proc. R. Soc. A 209, 248 (1951); W. G. Dixon, Dynamics of extended bodies in general relativity. I. Momentum and angular momentum, Proc. R. Soc. A 314, 499 (1970).

[37] See Supplemental Material at http://link.aps.org/ supplemental/10.1103/PhysRevLett.128.011101 for a collection of the relevant Feynman rules, details on the integrations and the total radiated angular momentum and 
energy. In addition we present the full expressions for (19) and (20) in an ancillary file.

[38] E. Herrmann, J. Parra-Martinez, M. S. Ruf, and M. Zeng, Gravitational Bremsstrahlung from Reverse Unitarity, Phys. Rev. Lett. 126, 201602 (2021).

[39] E. Herrmann, J. Parra-Martinez, M. S. Ruf, and M. Zeng, Radiative classical gravitational observables at $\mathcal{O}\left(\mathcal{G}^{3}\right)$ from scattering amplitudes, J. High Energy Phys. 10 (2021) 148.

[40] D. A. Kosower, B. Maybee, and D. O'Connell, Amplitudes, observables, and classical scattering, J. High Energy Phys. 02 (2019) 137.

[41] B. Maybee, D. O'Connell, and J. Vines, Observables and amplitudes for spinning particles and black holes, J. High Energy Phys. 12 (2019) 156.

[42] P. Di Vecchia, C. Heissenberg, R. Russo, and G. Veneziano, Universality of ultra-relativistic gravitational scattering, Phys. Lett. B 811, 135924 (2020).

[43] T. Damour, Radiative contribution to classical gravitational scattering at the third order in $G$, Phys. Rev. D 102, 124008 (2020).

[44] Z. Bern, L. J. Dixon, D. C. Dunbar, and D. A. Kosower, One loop $\mathrm{n}$ point gauge theory amplitudes, unitarity and collinear limits, Nucl. Phys. B425, 217 (1994); Fusing gauge theory tree amplitudes into loop amplitudes, Nucl. Phys. B435, 59 (1995); R. Britto, F. Cachazo, and B. Feng, Generalized unitarity and one-loop amplitudes in $N=4$ super-YangMills, Nucl. Phys. B725, 275 (2005).

[45] Z. Bern, J. J. M. Carrasco, and H. Johansson, New relations for gauge-theory amplitudes, Phys. Rev. D 78, 085011 (2008); Z. Bern, J. J. M. Carrasco, and H. Johansson, Perturbative Quantum Gravity as a Double Copy of Gauge Theory, Phys. Rev. Lett. 105, 061602 (2010); Z. Bern, J. J. M. Carrasco, L. J. Dixon, H. Johansson, and R. Roiban, Simplifying multiloop integrands and ultraviolet divergences of gauge theory and gravity amplitudes, Phys. Rev. D 85, 105014 (2012); Z. Bern, J. J. M. Carrasco, W.-M. Chen, H. Johansson, R. Roiban, and M. Zeng, Five-loop four-point integrand of $N=8$ supergravity as a generalized double copy, Phys. Rev. D 96, 126012 (2017); Z. Bern, J. Joseph Carrasco, W.-M. Chen, A. Edison, H. Johansson, J. ParraMartinez, R. Roiban, and M. Zeng, Ultraviolet properties of $\mathcal{N}=8$ supergravity at five loops, Phys. Rev. D 98, 086021 (2018); Z. Bern, J. Joseph Carrasco, M. Chiodaroli, H. Johansson, and R. Roiban, The duality between color and kinematics and its applications, arXiv:1909.01358.

[46] Z. Bern, C. Cheung, R. Roiban, C.-H. Shen, M. P. Solon, and M. Zeng, Black hole binary dynamics from the double copy and effective theory, J. High Energy Phys. 10 (2019) 206.

[47] J. Parra-Martinez, M. S. Ruf, and M. Zeng, Extremal black hole scattering at $\mathcal{O}\left(G^{3}\right)$ : Graviton dominance, eikonal exponentiation, and differential equations, J. High Energy Phys. 11 (2020) 023.

[48] Z. Bern, J. Parra-Martinez, R. Roiban, M. S. Ruf, C.-H. Shen, M. P. Solon, and M. Zeng, Scattering Amplitudes and Conservative Binary Dynamics at $\mathcal{O}\left(G^{4}\right)$, Phys. Rev. Lett. 126, 171601 (2021)

[49] C. Dlapa, G. Kälin, Z. Liu, and R. A. Porto, Dynamics of binary systems to fourth post-Minkowskian order from the effective field theory approach, arXiv:2106.08276.
[50] Z. Bern, C. Cheung, R. Roiban, C.-H. Shen, M. P. Solon, and M. Zeng, Scattering Amplitudes and the Conservative Hamiltonian for Binary Systems at Third Post-Minkowskian Order, Phys. Rev. Lett. 122, 201603 (2019);

[51] C. Cheung and M. P. Solon, Classical gravitational scattering at $\mathcal{O}\left(\mathrm{G}^{3}\right)$ from Feynman diagrams, J. High Energy Phys. 06 (2020) 144; G. Kälin, Z. Liu, and R. A. Porto, Conservative Dynamics of Binary Systems to Third PostMinkowskian Order from the Effective Field Theory Approach, Phys. Rev. Lett. 125, 261103 (2020).

[52] P. Di Vecchia, C. Heissenberg, R. Russo, and G. Veneziano, The eikonal approach to gravitational scattering and radiation at $\mathcal{O}\left(\mathcal{G}^{3}\right)$, arXiv:2104.03256; N. E. J. Bjerrum-Bohr, P. H. Damgaard, L. Planté, and P. Vanhove, The amplitude for classical gravitational scattering at third post-Minkowskian order, arXiv:2105.05218.

[53] D. Bini, T. Damour, and A. Geralico, Scattering of tidally interacting bodies in post-Minkowskian gravity, Phys. Rev. D 101, 044039 (2020); C. Cheung and M. P. Solon, Tidal Effects in the Post-Minkowskian Expansion, Phys. Rev. Lett. 125, 191601 (2020); K. Haddad and A. Helset, Tidal effects in quantum field theory, J. High Energy Phys. 12 (2020) 024; G. Kälin, Z. Liu, and R. A. Porto, Conservative tidal effects in compact binary systems to next-to-leading post-Minkowskian order, Phys. Rev. D 102, 124025 (2020); A. Brandhuber and G. Travaglini, On higher-derivative effects on the gravitational potential and particle bending, J. High Energy Phys. 01 (2020) 010; M. Accettulli Huber, A. Brandhuber, S. De Angelis, and G. Travaglini, Note on the absence of $R^{2}$ corrections to Newton's potential, Phys. Rev. D 101, 046011 (2020); M. Accettulli Huber, A. Brandhuber, S. De Angelis, and G. Travaglini, Eikonal phase matrix, deflection angle and time delay in effective field theories of gravity, Phys. Rev. D 102, 046014 (2020); Z. Bern, J. Parra-Martinez, R. Roiban, E. Sawyer, and C.-H. Shen, Leading nonlinear tidal effects and scattering amplitudes, J. High Energy Phys. 05 (2021) 188; C. Cheung, N. Shah, and M. P. Solon, Mining the geodesic equation for scattering data, Phys. Rev. D 103, 024030 (2021).R. Aoude, K. Haddad, and A. Helset, Tidal effects for spinning particles, J. High Energy Phys. 03 (2021) 097.

[54] D. Amati, M. Ciafaloni, and G. Veneziano, Higher order gravitational deflection and soft bremsstrahlung in planckian energy superstring collisions, Nucl. Phys. B347, 550 (1990); P. Di Vecchia, A. Luna, S. G. Naculich, R. Russo, G. Veneziano, and C. D. White, A tale of two exponentiations in $\mathcal{N}=8$ supergravity, Phys. Lett. B 798, 134927 (2019); P. Di Vecchia, S. G. Naculich, R. Russo, G. Veneziano, and C. D. White, A tale of two exponentiations in $\mathcal{N}=8$ supergravity at subleading level, J. High Energy Phys. 03 (2020) 173; Z. Bern, H. Ita, J. Parra-Martinez, and M. S. Ruf, Universality in the Classical Limit of Massless Gravitational Scattering, Phys. Rev. Lett. 125, 031601 (2020); M. Accettulli Huber, A. Brandhuber, S. De Angelis, and G. Travaglini, From amplitudes to gravitational radiation with cubic interactions and tidal effects, arXiv:2012.06548; P. Di Vecchia, C. Heissenberg, R. Russo, and G. Veneziano, Radiation reaction from soft theorems, arXiv:2101.05772; Y. Fabian Bautista and A. Guevara, From scattering amplitudes to classical physics: Universality, double copy and 
soft theorems, arXiv:1903.12419; A. Laddha and A. Sen, Gravity waves from soft theorem in general dimensions, J. High Energy Phys. 09 (2018) 105; A. Laddha and A. Sen, Logarithmic terms in the soft expansion in four dimensions, J. High Energy Phys. 10 (2018) 056; B. Sahoo and A. Sen, Classical and quantum results on logarithmic terms in the soft theorem in four dimensions, J. High Energy Phys. 02 (2019) 086; A. Laddha and A. Sen, Classical proof of the classical soft graviton theorem in $D>4$, Phys. Rev. D 101, 084011 (2020); A. Priya Saha, B. Sahoo, and A. Sen, Proof of the classical soft graviton theorem in $D=4$, J. High Energy Phys. 06 (2020) 153; Manu A, D. Ghosh, A. Laddha, and P. V. Athira, Soft radiation from scattering amplitudes revisited, arXiv:2007.02077; B. Sahoo,
Classical sub-subleading soft photon and soft graviton theorems in four spacetime dimensions, J. High Energy Phys. 12 (2020) 070.

[55] T. Damour, Classicaland quantum scattering in postMinkowskian gravity, Phys. Rev. D 102, 024060 (2020); N. E. J. Bjerrum-Bohr, A. Cristofoli, and P. H. Damgaard, Post-Minkowskian scattering angle in Einstein gravity, J. High Energy Phys. 08 (2020) 038; N. Emil, J. BjerrumBohr, P. H. Damgaard, L. Planté, and P. Vanhove, Classical gravity from loop amplitudes, arXiv:2104.04510; G. Kälin and R. A. Porto, From boundary data to bound states, J. High Energy Phys. 01 (2020) 072; From boundary data to bound states. Part II. Scattering angle to dynamical invariants (with twist), J. High Energy Phys. 02 (2020) 120. 